\title{
Fever of unknown origin following parathyroidectomy prior to onset of typical polymyalgia rheumatica symptoms: a case report
}

This article was published in the following Dove Press journal: International Journal of General Medicine

\author{
Akinori Kanzaki ${ }^{1,2}$ \\ Kiyoshi Matsui ${ }^{3}$ \\ Tadahiko Sukenaga ${ }^{1,2}$ \\ Koushi Mase' \\ Aya Nishioka' \\ Tomoharu Tamori ${ }^{4}$ \\ Seiko Kataoka' \\ Hiroyuki Konya' \\ Shin Mizutani ${ }^{4}$ \\ Akira Takeda' \\ Masato Koseki' \\ Tetsuo Nishiura' \\ Hidenori Koyama ${ }^{2}$ \\ Hajime Sano 3
}

'Department of Internal Medicine, Ashiya Municipal Hospital, Ashiya, Hyogo, Japan; ${ }^{2}$ Division of Diabetes, Endocrinology and Metabolism, Department of Internal Medicine, Hyogo College of Medicine,

Nishinomiya, Hyogo, Japan; ${ }^{3}$ Division of Rheumatology, Department of Internal Medicine, Hyogo College of Medicine, Nishinomiya, Hyogo, Japan; ${ }^{4}$ Department of Surgery, Ashiya Municipal Hospital, Ashiya, Hyogo, Japan
Correspondence: Akinori Kanzaki Department of Internal Medicine, Ashiya Municipal Hospital, 39-I Asahigaoka-cho, Ashiya, Hyogo 659-8502, Japan

Tel +8I 797312156

Fax +8I 797228822

Email aknewendocrine 1984I223@gmail. com

\begin{abstract}
Polymyalgia rheumatica (PMR) is a disease commonly seen in elderly individuals, however, the etiology has not been reported. Typical clinical features include bilateral shoulder pain and morning stiffness, while serologic autoantibody test findings are negative. Approximately $40 \%-50 \%$ of affected patients present with low-grade fever, fatigue, and appetite loss, which we often experience in the field of general medicine, and thus, the condition should not be given low priority. However, knowledge regarding such constitutional manifestations is also limited. We encountered an elderly woman with a fever of unknown origin that developed following a parathyroidectomy for a single parathyroid adenoma, after which severe shoulder pain and morning stiffness emerged, leading to a diagnosis of PMR. The fever developed several days prior to appearance of severe pain, which is an uncommon presentation in PMR cases. Our patient had low-grade inflammation without pyrexia prior to the surgery, which might have been an important reason for the accelerated immoderate immune activation leading to PMR induced by surgery in this case. Furthermore, she was infected with the influenza A virus 3 weeks before coming to us. Some reports have suggested a relationship between the influenza virus or vaccine and PMR. It is difficult to conclude regarding the definite trigger in our patient, though the details of this case should be helpful for a better understanding of the disease.
\end{abstract}

Keywords: polymyalgia rheumatica, fever of unknown origin, immoderate immune activation, parathyroidectomy, influenza A viral infection, single parathyroid adenoma

\section{Introduction}

Polymyalgia rheumatica (PMR) is a chronic inflammatory disease commonly seen in elderly individuals. Typical clinical features are bilateral shoulder pain and morning stiffness, while serologic autoantibody test results are negative. Approximately $40 \%-50 \%$ of affected patients are presented with low-grade fever, fatigue, and appetite loss, ${ }^{1}$ which we often experience in the field of general medicine, and thus, the condition should not be given low priority. However, knowledge regarding such constitutional manifestations is limited, partly because the pathophysiology of the disease has not been revealed.

Genetic and environmental factors are thought to be pathogenetic components, with HLA-DRB1*04 class II alleles a possible influence. ${ }^{2}$ As for environmental factors, infectious agents such as Mycoplasma pneumoniae, Chlamydophila pneumoniae, and Human herpesvirus 3 may also have an association with the disease etiology. ${ }^{3,4}$ Nevertheless, though such findings are noteworthy, the mechanism remains poorly understood.

The patient in the present study was a 67-year-old woman with primary hyperparathyroidism, who had been infected with influenza A virus and suffered from an

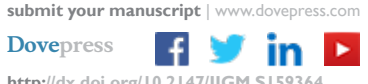


upper respiratory tract infection (URTI), then developed PMR after undergoing a parathyroidectomy procedure. Notably, a high fever developed on postoperative day 2 without other complications including infection, after which severe bilateral shoulder pain and morning stiffness were noted. Approximately $40 \%-50 \%$ of PMR patients are presented with low-grade fever, though it is unusual that a fever of unknown origin emerged for several days prior to the typical complication of severe pain and, to the best of our knowledge, this is the first such case reported. In addition, we present the clinical progress in detail for better elucidation of PMR.

\section{Case report}

A 67-year-old woman diagnosed with primary hyperparathyroidism and a medical history of fatty liver was hospitalized in February 2017 to undergo a parathyroidectomy for a well-defined flat single parathyroid adenoma in the left lobe that measured $15 \mathrm{~mm}$. There was no family history of hypercalcemia. Although she had been infected with the influenza A virus 3 weeks prior and suffered from an URTI several days before, vital signs and physical findings were not remarkable on the day of admission. Initial blood test results revealed features of hyperparathyroidism, a high concentration of C-reactive protein (CRP), anemia, and liver function disorder, including the following measurements: white blood cell (WBC) count $6.3 \times 10^{9} / \mathrm{L}$, hemoglobin $94 \mathrm{~g} / \mathrm{L}$, platelets $374 \times 10^{9} / \mathrm{L}$, albumin $31 \mathrm{~g} / \mathrm{L}, \mathrm{CRP} 68.5 \mathrm{mg} / \mathrm{L}$, aspartate aminotransferase $29 \mathrm{U} / \mathrm{L}$, alanine aminotransferase $69 \mathrm{U} / \mathrm{L}$, alkaline phosphatase $1015 \mathrm{U} / \mathrm{L}, \mathrm{\gamma}$-glutamyl transferase 197 $\mathrm{U} / \mathrm{L}$, creatine kinase $15 \mathrm{U} / \mathrm{L}$, amylase $53 \mathrm{U} / \mathrm{L}$, creatinine $47.7 \mu \mathrm{mol} / \mathrm{L}$, blood urea nitrogen $4.7 \mathrm{mmol} / \mathrm{L}$, sodium $(\mathrm{Na})$ $143 \mathrm{mmol} / \mathrm{L}$, potassium (K) $4.0 \mathrm{mmol} / \mathrm{L}$, chlorine $(\mathrm{Cl}) 108$ $\mathrm{mmol} / \mathrm{L}$, calcium (Ca) $3.02 \mathrm{mmol} / \mathrm{L}$, phosphorus (P) 0.94 $\mathrm{mmol} / \mathrm{L}$, intact parathyroid hormone $16.2 \mathrm{pmol} / \mathrm{L}$, parathyroid hormone-related protein $<1.1 \mathrm{pmol} / \mathrm{L}$, and $1,25-(\mathrm{OH})_{2}$ vitamin D $242.4 \mathrm{pmol} / \mathrm{L}$.

For surgery, the approach was started with a $4 \mathrm{~cm}$ lateral incision. Bleeding totaled $3 \mathrm{~mL}$ and the procedure was performed without any complications, after which hypercalcemia showed remission. However, a moderate fever developed 2 days after surgery (Figure 1), though there were no other symptoms such as fatigue, pain, or physical findings including surgery scar redness, joint swelling, or eruption. Body temperature never exceeded $38^{\circ} \mathrm{C}$ and the greatest fluctuation was within $1^{\circ} \mathrm{C}$. Computed tomography (CT) scanning performed just prior to surgery revealed no remarkable findings, such as tuberculosis or abscess. Although the operation was a relatively minor procedure, the fever was considered to be the result of the healing process and the patient continued to be followed. When given acetaminophen as rescue, the fever declined, though emerged again and rose to $38^{\circ} \mathrm{C}$ on postoperative day 6 (Figure 1). Furthermore, blood test results showed that a moderately high concentration of CRP $(79.8 \mathrm{mg} / \mathrm{L})$ remained, though there was no increase in WBC (Table 1). For symptomatic treatment, daily administrations of acetaminophen were started, which controlled the fever.

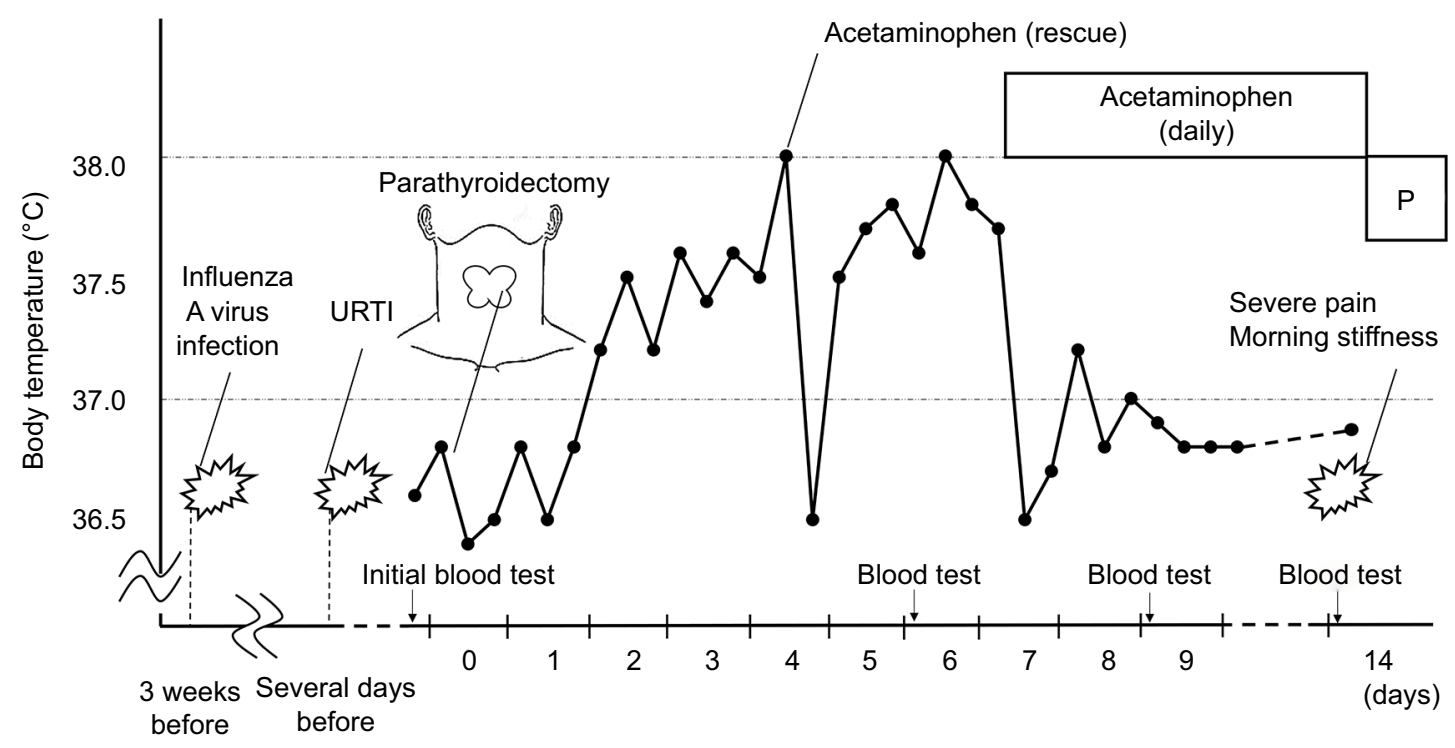

Figure I Course of body temperature and clinical events.

Notes: Pyrexia was not seen on the day of admission. On postoperative day 2, body temperature suddenly rose. Thereafter, pyrexia was controlled with daily administrations of acetaminophen. Three days after discharge, severe pain in both shoulders and morning stiffness suddenly occurred. Body temperature was controlled by daily acetaminophen administration and remained normal after switching to prednisolone.

Abbreviation: P, prednisolone; URTI, upper respiratory tract infection. 
Table I Blood test findings prior to hospitalization and various time points after admission

\begin{tabular}{|c|c|c|c|c|c|c|c|}
\hline Investigation & I month before & Admission & PD 6 & PD 9 & PD I4 & PD 21 & PD 28 \\
\hline WBC $\left(\times 10^{2} / L\right)$ & 5.28 & 6.30 & 5.50 & 6.14 & 7.66 & 8.58 & 12.99 \\
\hline CRP $(\mathrm{mg} / \mathrm{L})$ & 0.7 & 68.5 & 79.8 & 52.1 & 112.4 & 1.7 & 0.1 \\
\hline $\operatorname{ESR}(\mathrm{mm} / \mathrm{h})$ & NT & NT & NT & 113 & NT & 36 & 3 \\
\hline
\end{tabular}

Notes: A blood examination, including CRP, ESR, and WBC, was performed I month prior to hospitalization and again at various time points after admission. Increased levels of CRP with no increase in WBC were noted on the day of admission, and then worsened. However, after starting prednisolone on PD I4, the levels of CRP and ESR showed dramatic decreases.

Abbreviations: CRP, C-reactive protein; ESR, erythrocyte sedimentation rate; NT, not tested; PD, postoperative day; WBC, white blood cell.

To investigate further, screening for collagen disease including vasculitis was performed, including erythrocyte sedimentation rate (ESR), rheumatoid factor, anti-cyclic citrullinated peptide antibody, antinuclear antibody, serum complement titer, antineutrophil cytoplasmic antibody, and serum ferritin. Thereafter, the patient was discharged on postoperative day 10 and asked to return on an outpatient basis. Surprisingly, pain in both shoulders and morning stiffness lasting for $>1$ hour suddenly appeared 3 days after discharge. The pain was too severe to sit up, and thus, the patient returned to our hospital. Our examination found no evidence of eruption, peripheral pitting edema, or tumor in a temporal artery. Furthermore, a screening blood test for collagen disease performed before discharge was negative (data not shown), except for ESR $(113 \mathrm{~mm} / \mathrm{h})$, though an elevated concentration of CRP $(112.4 \mathrm{mg} / \mathrm{L})$ and high level of ESR $(101 \mathrm{~mm} / \mathrm{h})$ were noted (Table 1$)$. A CT scan did not reveal any inflammatory findings including vasculitis. Based on the symptoms and blood test results, though without joint sonography findings, the patient had $>4$ points in the American College of Rheumatology and European League Against Rheumatism classification criteria and disease activity as assessed by PMR activity score was $>17$ points. ${ }^{5,6}$ Therefore, we made a diagnosis of PMR and low-dose prednisolone was started, after which the symptoms rapidly improved and inflammation was reduced within a few days (Table 1). Body temperature, which had been under control with acetaminophen, remained controlled after switching to prednisolone. After 1 year of treatment, the dose of prednisolone was successfully reduced in February 2018.

Written informed consent was obtained from the patient to use anonymized information from their medical records and the CT scan and laboratory test results. The patient granted permission for the publication of all anonymized information.

\section{Discussion}

The present patient was an elderly woman with hypercalcemia, who developed a fever of unknown origin after undergoing a parathyroidectomy for a single parathyroid adenoma.
Following that procedure, severe shoulder pain and morning stiffness emerged, and she was diagnosed with PMR. She had been infected with the influenza A virus and suffered from a URTI prior to admission, while vital signs were not remarkable before surgery. We made a clinical diagnosis of PMR based on sudden onset of pain in both shoulders and morning stiffness without swelling of the temporal artery or other physical findings, as well as age over 50 years, abnormal CRP and ESR, and negative for serologic antibodies. ${ }^{5}$ In addition, the good effect of steroid treatment and no abnormal CT findings were considered to support that diagnosis. It is widely accepted that $\sim 40 \%-50 \%$ of PMR patients have constitutional symptoms such as low-grade fever, though many details remain unknown. Our patient developed a fever $>10$ days prior to the appearance of the typical complication of severe pain, which is an uncommon presentation in PMR cases. Although we initially considered the impact of a surgery-related infection, the patient did not show any swelling, pain, or heat in the scar area. Thus, we next examined for resorption fever, as she underwent a parathyroidectomy just 2 days prior to the appearance of pyrexia. A surgical injury can certainly cause fever, though we do not think that the present case can be simply regarded as a consequence of the progress of healing following the operation, because pyrexia did not decline until initiation of daily acetaminophen in spite of the minor surgery procedure performed without complications. Moreover, neither joint swelling nor eruption, which would suggest apparent collagen disease, was found and serologic autoantibody tests were negative. In addition, the situation was clearly not a drug-induced fever.

Fever is partly responsible for elevated secretion of interleukin 1 (IL-1) and IL-6, which raise the body temperature set point mediated by prostaglandin E2. It is also widely known that these cytokines activate monocytes, macrophages, dendritic cells (DCs), and lymphocytes, while some types of surgery procedures, such as cholecystectomy and elective hysterectomy, can increase the number of circulating blood DCs, which stimulate lymphocyte immune response in humans. ${ }^{7}$ Furthermore, thyroid epithelial cell necrosis has 
been shown to provoke maturation of intrathyroidal DCs and initiation of autoimmune response in mice. ${ }^{8}$ Thus, a surgical tissue injury including that in the neck area may cause activation of DCs by releasing cytokines, such as IL-1 or IL-6, and other cellular products. Activated DCs induce polarization of CD4 T cells into Th17 cells, resulting in IL-17 in plasma produced by Th17, and that along with co-stimulus with IL-6 can promote production of IL-6, which is thought to be a key cytokine in the pathogenesis of giant cell arteritis and PMR. ${ }^{9-11}$ Together, these findings support our speculation that immoderate immune activation was triggered by parathyroidectomy and caused pyrexia in our patient.

It is quite difficult to discuss in detail how a parathyroidectomy, a minor operation, caused immoderate immune activation in our patient. Nevertheless, a previous case report noted a lasting high fever of undetermined origin in a patient lacking typical shoulder joint pain that was due to PMR. ${ }^{12}$ That important case demonstrated that low-grade fever does not exclude a diagnosis of PMR, in other words, low-grade inflammation might be a foundation of immune system collapse under some unknown factors related to its etiology, such as a genetic factor. Previous reports have confirmed that PMR patients with polymorphisms in the promotor of the IL- 6 gene have a higher risk of developing relapse or recurrence, and show a persistently increased serum concentration of IL- $6 .{ }^{13}$ This may also indicate that a high level of IL-6 partially influences onset. Therefore, we speculate that the moderate level of CRP prior to surgery in our patient indicated the existence of increased IL-6, which partly accelerated the disturbance.

Infectious agents are also known to be triggers of PMR. A previous case report noted an elderly Japanese woman who developed PMR several days after developing an influenza B virus infection. ${ }^{14}$ Our patient does not closely match that previous report, because she was infected with the influenza A virus 3 weeks prior to admission and vital signs were not remarkable until pyrexia emerged. On the other hand, attention should be paid to another study in which the relationship between influenza vaccine and PMR was analyzed. ${ }^{15}$ According to those findings, administration of influenza vaccine can cause PMR within several weeks or up to 3 months. Our patient did not receive an influenza vaccine, though we cannot completely deny a relationship, because a vaccine is produced from the virus itself. Nevertheless, it is quite reasonable to consider that the moderate level of CRP on the day of admission was due to a URTI that developed several days prior, because the condition of our patient was generally good, without any findings in the initial CT scan and CRP measured 1 month before was not abnormal (Table 1). Moreover, 3 weeks had passed since infection with influenza A. Thus, we could not conclude any other factors except for URTI for the elevated CRP.

\section{Conclusion}

We experienced a case of PMR, in which pyrexia preceded shoulder pain and morning stiffness, which developed following a parathyroidectomy procedure for a single parathyroid adenoma with hypercalcemia in an elderly woman who had recently been infected with influenza $A$ virus and suffered from URTI just prior to surgery. Although several studies have examined the mechanism of PMR onset, that has yet to be clarified. It is also difficult to conclude regarding the definite trigger in our case, though we consider that the moderate level of CRP before surgery might have provided a foundation to accelerate immoderate immune activation leading to PMR caused by a parathyroidectomy procedure. Nevertheless, as we only have findings of a single patient, additional investigation is needed. We believe that the details of this case will contribute to revealing the etiology of this complex and important disease.

\section{Acknowledgment}

We appreciate Mark Benton for providing native-level English language support.

\section{Disclosure}

The authors report no conflicts of interest in this work.

\section{References}

1. Masson C, Gonzalez-Gay MA. Polymyalgia Rheumatica and Giant Cell Arteritis. EULAR Textbook on Rheumatic Disease, 2nd ed. London: BMJ Publishing Group; 2015:754-778.

2. Gonzalez-Gay MA, Amoli MM, Garcia-Porrua C, et al. Genetic markers of disease susceptibility and severity in giant cell arteritis and polymyalgia rheumatica. Semin Arthritis Rheum. 2003;33:38-48.

3. Elling P, Olsson AT, Elling H. Synchronous variations of the incidence of temporal arteritis and polymyalgia rheumatica in different regions of Denmark; association with epidemics of Mycoplasma pneumoniae infection. J Rheumatol. 1996;23:112-119.

4. Rheel RL, Grayson PC, Merkel PA, Tomasson G. Infectious and the risk of incident giant cell arteritis: a population-based, case-control study. Ann Rheum Dis. 2017;76:1031-1035.

5. Dasgupta B, Cimmino MA, Kremers HM, et al. 2012 provisional classification criteria for polymyalgia rheumatica: a European League Against Rheumatism/ American College of Rheumatology collaborative initiative. Arthritis Rheum. 2012;64:943-954.

6. Leeb BF, Bird HA. A disease activity score for polymyalgia rheumatica. Ann Rheum Dis. 2004;10:1279-1283.

7. Ho CS, López JA, Vuckovic S, Pyke CM, Hockey RL, Hart DN. Surgical and physical stress increases circulating blood dendritic cell counts independently of monocyte counts. Blood. 2001;98:140-145.

8. Li HS, Verginis P, Carayanniotis G. Maturation of dendritic cells by necrotic thyrocytes facilitates induction of experimental autoimmune thyroiditis. Clin Exp Immunol. 2006;144:467-474.

9. Nesher G, Breuer GS. Giant cell arteritis and polymyalgia rheumatica: 2016 update. Rambam Maimonides Med J. 2016;7:e0035. 
10. Samson M, Audia S, Fraszczak J, et al. Th1 and Th17 lymphocytes expressing CD161 are implicated in giant cell arteritis and polymyalgia rheumatica pathogenesis. Arthritis Rheum. 2012;64: 3788-3798.

11. Ogura H, Murakami M, Okuyama Y, et al. Interleukin-17 promotes autoimmunity by triggering a positive-feedback loop via interleukin-6 induction. Immunity. 2008;29:628-636.

12. Al Mahdy H, Martindale S. Polymyalgia rheumatica presenting as nocturnal fever of undetermined origin. JRSM Short Rep. 2010;1:11.
13. Boiardi L, Casali B, Farnetti E, et al. Relationship between interleukin 6 promoter polymorphism at position -174 , IL- 6 serum levels, and the risk of relapse/recurrence in polymyalgia rheumatica. J Rheumatol. 2006;33:703-708.

14. Iwata K, Mizuno Y. A case of polymyalgia rheumatica following influenza B infection. Int J Gen Med. 2015;8:345-347.

15. Soriano A, Verrecchia E, Marinaro A, et al. Giant cell arteritis and polymyalgia rheumatica after influenza vaccination: report of 10 cases and review of the literature. Lupus. 2012;21:153-157.
International Journal of General Medicine

\section{Publish your work in this journal}

The International Journal of General Medicine is an international, peer-reviewed open-access journal that focuses on general and internal medicine, pathogenesis, epidemiology, diagnosis, monitoring and treatment protocols. The journal is characterized by the rapid reporting of reviews, original research and clinical studies across all disease areas.

\section{Dovepress}

The manuscript management system is completely online and includes a very quick and fair peer-review system, which is all easy to use. Visit http://www.dovepress.com/testimonials.php to read real quotes from published authors.

Submit your manuscript here: https://www.dovepress.com/international-journal-of-general-medicine-journal 